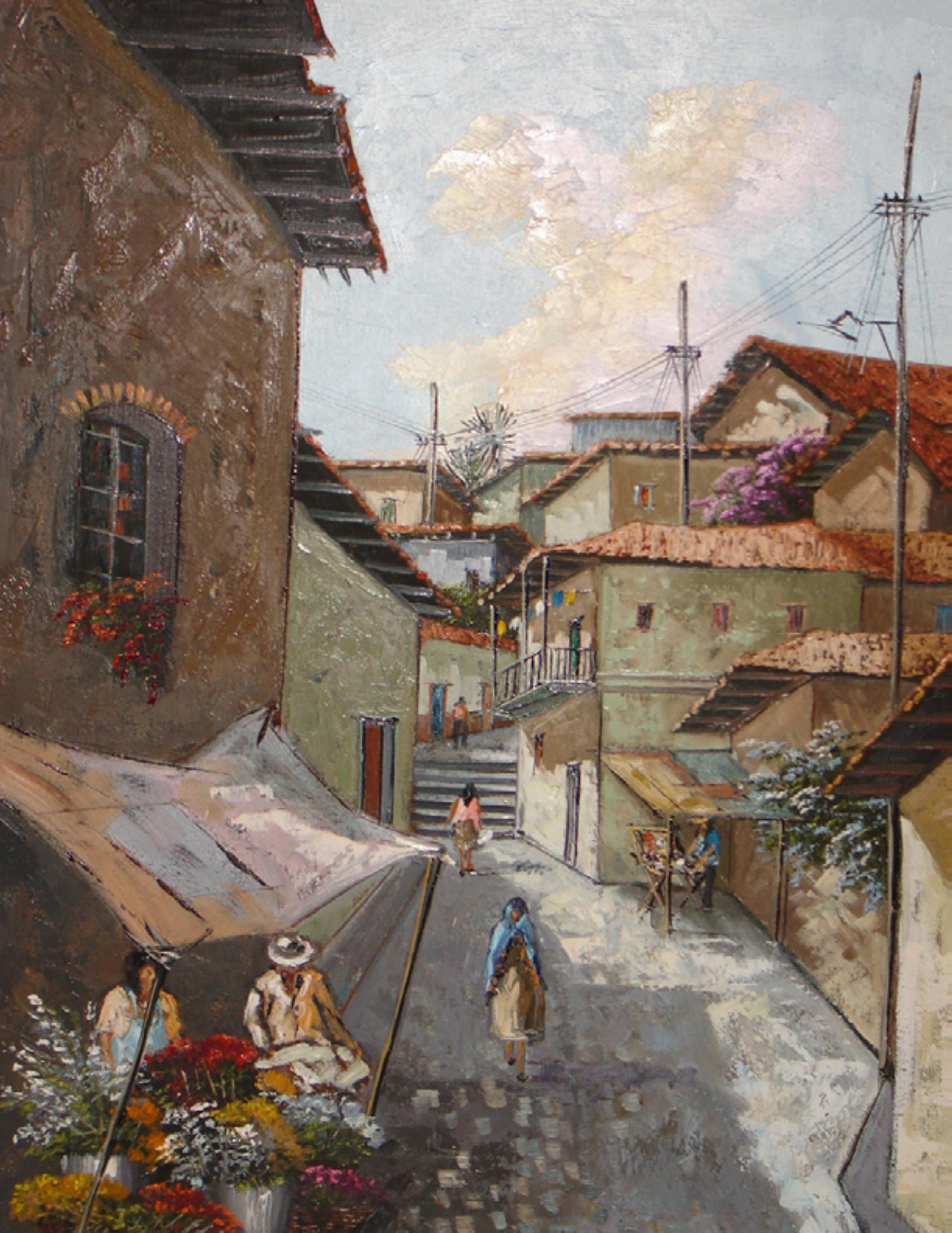




\title{
3 la historiografía sobre ciudades, regiones y urbanización en Honduras: apuntes y bibliografía mínima
}

\author{
DARÍO A. EURAQUE
}

La historiografía sobre poblamiento, territorialidad, urbanización y movimientos demográficos y sus implicaciones sociales y culturales en Honduras es mínima, especialmente previo a la década de 1950 del siglo pasado, y en particular los padrones y cuadros estadísticos decimonónicos, los cuales aun carecen de análisis históricos serios (Avalos y Pagan Solórzano `97). Se pudiese decir lo mismo sobre las ultimas cinco décadas, con la diferencia de que para el periodo de 1950 a 2000 existe una especie de sociología histórica hondureña sobre la urbanización moderna (Salomón y Galo 1988) fundamentada mas que todo en datos censales de población, migración (Sierra Fonseca 2004) y vivienda (Sierra '82), o en algunos casos, mediante encuestas sobre problemas particulares como la pobreza, la salud publica, el sistema educacional o semejantes (Menjivar y Zelaya 2004; Figueroa y Trejo 2004).

Estos estudios se prestan para desglosar a grandes rasgos la infraestructura de la hondureñidad moderna en el Siglo XX (Euraque 03). Como comentáremos mas adelante, aunque valiosos, estos estudios poco nos trasmiten sobre la vida cotidiana y los imaginarios urbanos modernos; se esmeran mas por ofrecernos datos macros y sociológicos (Gibson, ` 70); Martinson, ’ 70) y Del Cid, ‘ 88). De hecho, hoy en día hasta contamos con estudio de cambios poblacionales y su impacto ecológico estudiados por satélites (Dodds 2004). Para trascender y estos y estudios semejantes, aun tenemos que recurrir, como carecemos de una historia social y cultural urbana, a ciertas contribuciones de una muy efímera historiografía fotográfica y arquitectónica y novelesca, la cual apenas existe para Tegucigalpa y San Pedro Sula, como lo señalaremos pronto.

Ahora bien, durante el Siglo XX, algunos eminentes historiadores le dieron seguimiento a la tradición de los cronistas españoles (Carias '98) en cuanto un esfuerzo por relatar, exaltar, y narrar la fundación y pasado de ciudades y villas coloniales importantes, especialmente Gracias a Dios, Comayagua, Tegucigalpa y otras vinculadas con lo que Leticia Oyuela llama "el esplendor y miseria de la minería en Honduras.” (Duron, 1904; Bobadilla `36; Lunardi, 1946; Raudales, 1950; y Reina Valenzuela, 1951, 1968, 1981). Durante el Siglo XX las diferentes publicaciones de la Sociedad Hondureña de Geografía e Historia, y su sucesora, la Academia Hondureña de Geografía e Historia, en sus diferentes épocas, han publicando gran cantidad de documentos sobre la fundación de ciudades coloniales en Honduras. Las publicaciones de ambas organizaciones fueron y siguen estando muy vinculados al Archivo Nacional de Honduras.

Otra tradición historiográfica semejante pero con mas interés en aspectos de la historia social y menos apegada a la exaltación colonial surgió después de las décadas de 1970 y 1980, aunque en su mayoría los autores desconocen entre si las contribuciones que hacen y por lo tanto se carece de dialogo intelectual que nutran los trabajos de uno y otros. Esta historiografía fragmentada amplió la variedad de ciudades y pueblos considerados, desde Jesús de Otoro, La Paz, La Ceiba, Olanchito, San Pedro Sula y Tela (Fiallos '70; Rivero y Morillo `78; Martínez Castillo '82; Machuca '83; Rendón Madrid, '85; González, '92; Euraque '92, '93; Posas, '93; Posas, '94; Oyuela '94; Funez, '95; Bueso Yescas, '96: Canelas Diaz, 99; Elvir, 2000; y Galeas, '01). Muchos de estos trabajos arrancan con los datos poblaciones aun vigentes recogidos por el gran historiador hondureño Antonio R. Vallejo (Vallejo 1893).

Una importante innovación historiográfica se genero simultáneamente con las tendencias registradas en el párrafo anterior y tiene su origen en problemas de la demografía colonial, la indígena especialmente, y que no se fijo hasta la publicación de Linda Newson en la década de 1980 (Newson '86 y '92). Nos referimos a la noción de la territorialidad y las poblaciones vinculadas con las ciudades y villas fundacionales pero que generan espacios diferentes, y de hecho regiones sociales y culturales no provistas en el régimen colonial. Innovador en este sentido fue la tesis de maestría de Rodolfo Pastor Fasquelle (`75), y contribuciones por catedráticos y estudiantes de la Carrera de Historia de la Universidad Nacional Autónoma de Honduras (Aguilar et al., 82; Mossi Sorto y Batres Galeano '82; Zelaya, '91, '95, y Chaverri, '92)

Vinculado a este esfuerzo por analizar la territorialidad a nivel general surgió también un interés por el pasado de ciertos municipios en un esfuerzo por especificar cambios regionales a través del tiempo. Ya para fines de la década de 1980 se publico por lo menos una visión general del pasado municipal en Honduras (Fiallos `89), mientras que otros autores escudriñaron municipios particulares a fines del Siglo XIX (Cruz Reyes et al, '80; Velez O. ‘82; Palacios '88; Infante et al., '88: Barahona et al., '89). Recientemente, el papel sociopolítico de la municipalidad entre fines de la colonia y mediados del Siglo XIX fue abordado a fondo en todo el contexto centroamericano, y el caso de Honduras también (Dym 2000). En ese contexto mas amplio merece también ubicarse anteriores y muy distinguidas contribuciones sobre la Alcaldía de Tegucigalpa previo y después de la colonia (Zelaya ` 92 y Oyuela `94; Barahona `96; Taracena `98; y Oyuela 2003). La capital colonial de Honduras, Comayagua, aun carece de este enfoque, aunque su arte y la urbanización si se ha abordado (Martínez Castillo `80).

Regiones y municipalidades para la época posterior a las guerras de la Independencia se analizaron sistemáticamente hace ya más de dos décadas en una tesis doctoral en EE.UU. que aun carece de traducción (Guevara- Escudero `83); existen también otros trabajos que se han aprovechado de la misma u otras obras para también delimitar 
la evolución de regiones geo-económicas en la historia de Honduras entre 1830 y 1930 y su relación con el desarrollo de las regiones bananeras (Euraque '92 y Herrera `93). Los últimos trabajos, cuyo enfoque particular es la costa caribeña de Honduras, en cierto sentido han sido superados o complementado por varias tesis doctorales que abordan muchos aspectos de la vida social y hasta ecológica de aquellas regiones (Soluri ‘98, Mack ‘2000; Tompson `2001, y (Payne `2005). Es mas, aun carecemos de una historiografía social de los asentamientos Garifunas, ello a pesar de las enormes contribuciones de William Davidson ( 76), y otros (Cruz Sandoval 2002 y Euraque 2004).

De nuevo, la mayoría de estos estudios hacen hincapié en población, territorialidad, economías y el papel del estado en estos contextos, sea el nacional o el municipal. Estos, como otros para el Siglo XX (Joya y Urquia ‘ 83 y Herrera ‘ 86 ); con escasas excepciones urbanas (Pastor Fasquelle `90) no comentaron a fondo la vida social. Con estas características para San Pedro Sula contamos ya con algunas memorias (Antunez Castillo, '67; Luque `79; Bueso Arias ‘91 y Herrera Bobadilla 2001), y otras obras que recurren a las imágenes fotográficas y la historia arquitectónica para ahondar en la vida cotidiana de San Pedro Sula en las primeras siete décadas del Siglo XIX (Stassano Raquel ' 97 y Escoto 2002). Sin embargo, para San Pedro Sula sigue sin llenarse el vacío que ya con la novela se comenzó a llenar la vida urbana en Tegucigalpa para el Siglo XX (Carias Zapata ' 80 y Duron ` 89 ).

En este contexto merecen también apreciarse las memorias recientes sobre uno de los barrios viejos de Tegucigalpa (Becerra 2004). Este genero goza de una larga tradición en Tegucigalpa que se remonta a comienzos hasta fines del Siglo XIX, especialmente con publicaciones de Ramón Rosa, Rafael Heliodoro Valle ( ‘54) y otros. Para el Siglo XX (Rosa '67), y que recientemente se recogió en una nueva edición de una obra seleccionada por el poeta Oscar Acosta publicada por primera vez en 1978 elogiando a Tegucigalpa, en sus 400 años de fundación (Acosta 2003). No existe obra semejante para San Pedro Sula, aunque si en sus 400 años de fundación se publicó una importante obra que luego se reeditó a fines del Siglo pasado (Bobadilla `36); también en este sentido cierto merito el esfuerzo personal del Profesor Ibrahim Gomero Idiaquez (' 93)

\section{BIBLIOGRAFÍA}

Antonio R. Vallejo, Primer Anuario Estadístico, Correspondiente Al Año de 1889 (Tegucigalpa: Editorial Universitario, [1893], 1997).

Rómulo E. Duron, La Provincia de Tegucigalpa bajo el Gobierno de Mallol (Tegucigalpa: Tipografía Nacional, 1904).

Nels Bengston, "Studies in the Geography of Honduras," Ph.D diss., Clark University, 1927.

Perfecto H. Bobadilla, Monografía Geográfica e Histórica de San Pedro Sula. IV Centenario de su Fundación, 1536-1936 (San Pedro Sula: Compañía Editora, 1936.

Federico Lunardi, La Fundación de la Ciudad de Gracias a Dios y de las Primeras Villas y Ciudades de Honduras (Tegucigalpa: s.n., 1946).

Ángel Raudales, Fundación, Desarrollo y Porvenir de San Pedro Sula (San Pedro Sula: s.n., 1950).

José Reina Valenzuela, "San Pedro Sula: Su Pasado, Su Presente, Su Porvenir," La Pajarita de Papel, (Oct.- Dic., 1951): 89-104.

Rafael Heliodoro Valle, "Tegucigalpa en 1855," en Ensayos Escogidos de Rafael Heliodoro Valle, Compilación, Roger Martínez Miralda y Enma Leticia Ordóñez San Martín (Tegucigalpa: Editorial Universitaria, [1954] 1991), pp. 149-152.

M. Antonio Rosa, La Tegucigalpa de Mis Primeros Años (Tegucigalpa: Imprenta Calderón, 1967. 1967.

Rubén Antúnez Castillo, Mis Memorias, Vol. 2. San Pedro Sula: Imprenta Suyapa,

José Reina Valenzuela, Comayagua Antañona, 1537-1821 (Tegucigalpa: Biblioteca de la Academia de Geografía e Historia, 1968). 1970).

José Nery Fiallos R. Apuntes Históricos [Sobre Jesús de Otoro] (Tegucigalpa, s.n.,

Jeffrey Gibson, "A Demographic Analysis of Urbanization: Evolution of a System of Cities in Honduras, El Salvador, and Costa Rica," Ph.D diss., Cornell Univsersity, 1970.

Ruth I. Shirey, "An Analysis of the Location of Manufacturing: Tegucigalpa and San Pedro Sula, Ph.D diss., University of Tennessee, 1970.

Tommy Martinson, "Selected Changes in Agricultural Production and Economic Rent Along the Western Highway of Honduras," Ph.D diss., University of Kansas, 1970. Gene Yeager, "Honduras Transportation and Communication Development: The Rise of Tegucigalpa, 1878-1900," Masters thesis, Tulane University, 1972.

William K. Crowley, "San Pedro Sula, Honduras: The Order And Disorder Of The Pubescent Period in Central America's Most Rapidly Growing City,” Ph.D diss., University of Oregon, 1972.

Kenneth Finney, "Precious Metal Mining and the Modernization of Honduras: In Quest of El Dorado (1880-1900)," Ph.D diss., Tulane University, 1973.

Hector Pérez Brignoli, "La Reforma Liberal en Honduras," en Cuadernos de Ciencias Sociales, No. 2 (Tegucigalpa: Editorial Nuevo Continente, 1973), pp. 1-86

Rodolfo Pastor Fasquelle, "Desarrollo Urbano en Honduras Colonial (15001650)," Master's Thesis, Tulane University, 1975.

Humberto Rivera y Morillo, San Pedro Usula: Génesis Histórica (San Pedro Sula: Central Impresora, 1978.

William V. Davidson, "Coastal Imperative Lost?: Village Abandonment Among the Honduran Garifuna," Actes du XLII Congres international Des Americanistes Vol. 6 (1979), pp. 571-576

Mauricio Castañeda Beuchot, Henecan: Historia de Un Golfo y un Puerto (Tegucigalpa: Tipografía López, 1979).

Gonzalo R. Luque, Memorias de un Sampedrano (San Pedro Sula: Impresora Hondureña, 1979)

Mario Felipe Martínez Castillo, "Desarrollo Urbano y Artístico de Comayagua durante la Colonia," Tesis Doctoral, Universidad de Sevilla, 1980.

Víctor C. Cruz Reyes, Perla Mossi de Carías, Annarella Vélez Osejo, Jorge Alberto Gálvez y Ricardo A. Urquía, "Yuscaran, 1880-1915," Tesis de Bachillerato, Carrera de Historia, UNAH, 1980.

Marcos Carias Zapata, Una Función con Móviles y Tentetiesos (Tegucigalpa: Editorial Guaymuras, 1980).

José Reina Valenzuela, Tegucigalpa: Sintesis Histórica, Tomo 1 (Tegucigalpa: Consejo Metropolitano del Distrito Central, 1981)

Rodrigo Fernández Vasquez, "Poblamiento y Ocupación Territorial en Centro América, 1870-1940," Estudios Sociales Centroamericanos, Año 10, No. 29 (Mayo- Agosto, 1981), pp. 143-214.

Manuel Aguilar, Marlene de Guardiola, Farlei Arita, y Sidalia Batres. "Dominio Territorial de los Ejidos de La Paz, Municipalidad de la Paz." Proyecto de Tesis de Bachillerato, Carrera de Historia, UNAH, 1982.

Perla Mossi Sorto, y Sidalia Batres Galeano, "Antecedentes Históricos Acerca De Dominio Territorial Urbano En Tegucigalpa (Siglo XIX).” Tesis de Licenciatura, Carrera de Historia, UNAH, 1982.

Annarela Vélez O., y Iván Herrera. "Historia De La Municipalidad De Tegucigalpa, Años 1870-1903.” Tesis de Licenciatura, Carrera de Historia, UNAH, 1982.

Mario F. Martínez Castillo, Apuntamientos para una Historia Colonial de Tegucigalpa y su Alcaldía Mayor (Tegucigalpa: Editorial Universitaria, 1982).

Olga Joya y Ricardo Urquía. "Incidencia Del Estado En El Desarrollo Económico De Tegucigalpa.” Tesis de Licenciatura, Carrera de Historia, UNAH, 1983.

Francisco Guevara-Escudero, "Nineteenth-Century Honduras: A Regional Approach To The Economic History of Central America, 1839-1914," Ph.D diss., New York University, 1983.

Alexis Machuca, La Paz: Semblanza Histórica de una Ciudad y Su Gente (Tegucigalpa: Secretaría de Cultura y Turismo, 1983)

Arturo Rendón Madrid, Santa Rosa de Copan: La Sultana de Occidente (Tegucigalpa: Secretaria de Turismo, 1985).

Linda Newson, The Conquest of Honduras: Indian Decline Under Spanish Rule (Boulder: Westview Press, 1986).

José Iván Herrera. "El Régimen Municipal y Los Enclaves," Economía Política, No. 24 (1986): 5-50

Leticia Salomón y Betenia Galo, "Expansión y Estructuración Urbana de Tegucigalpa [1880-1980],” en La Estructuración de las Capitales Centroamericanas, Rodrigo Fernández Vasquez y Mario Lungo, compiladores (San Jose: EDUCA, 1988)

Rafael Del Cid, "Populating a Green Desert: Population Policy and Development Their Effect on Population Redistribution. Honduras, 1876-1980," Ph.D diss., University of Texas- Austin, 1988.

Palacios et al., Carlos. "Evolución de la Tenencia de Tierra en el Municipio de Santa Rosa de Copán.” Tesis de Bachillerato, Carrera de Historia, UNAH 1988.

Segisfredo Infante, Ligia Page, Miriam Fernández, Stilita Flores Abrego y José Esteban Márquez. Presencia de Los Alemanes y de Otras Minorías Extranjeras en el Municipio de Choluteca (1900-1947). Tesis de Bachillerato, Carrera de Historia, UNAH, 1988.

Leticia Oyuela, Historian Minima de Tegucigalpa, Vista a Través de las Fiestas del Patrono San Miguel a partir de 1680 hasta finales del Siglo XIX (Tegucigalpa: Editorial Gua- 
ymuras, 1989)

Jose Barahona G., Luís A. Sánchez y José Darío Izaguirre. "La Evolución de la Propiedad Privada Terrateniente en el Municipio de Choluteca, 1864-1891," Tesis de Bachillerato, Carrera de Historia, UNAH, 1989. 1989).

Jorge Fidel Duron, El Barrio Encantado (Tegucigalpa: Editorial Universitaria, 1989).

Carmen Fiallos, Los Municipios de Honduras (Tegucigalpa: Editorial Universitaria,

Rodolfo Pastor Fasquelle, Biografía de San Pedro Sula: 1536-1954 (San Pedro Sula: Central Impresora, 1990).

José A. Sarmiento, Historia de Olancho (Tegucigalpa: Editorial Guaymuras, 1990).

Darío A. Euraque, "Merchants and Industrialists in Northern Honduras: The

Making of a National Bourgeoisie in Peripheral Capitalism, 1870s-1972," Ph.D diss.

University of Wisconsin- Madison, 1990.

Juan Angel Bueso Arias, Barro Criollo (San Pedro Sula: Centro Editorial, 1991).

Sucelinda Zelaya, Honduras: Proceso de Configuración Territorial (Tegucigalpa: s.n.,

1991, 1995)

Federico González. Paginas de Oriente [Danli] (Tegucigalpa: Litografía López, 1992).

Linda Newson, El Costo de la Conquista en Honduras (Tegucigalpa: Editorial Guaymuras, 1992).

Marielos Chaverri, La Formación Histórica de Honduras. Factores que Inciden en la Construcción de la Territorialidad (Tegucigalpa: Centro de Estudios Históricos y Sociales, 1992).

Darío A. Euraque, "Estructura Económica, Formación de Capital Industrial, Relaciones Familiares y Poder Político en San Pedro Sula: 1870s-1958," Revista Polémica, Costa Rica, No. 18 (Sept- Dic. 1992): 31-50.

Darío A. Euraque, "Zonas Regionales En La Formación Del Estado Hondureño, 1830s-1930s: El Caso De La Costa Norte," Revista Centroamericana de Economía, Tegucigalpa, Año 13, No. 39 (Sept.- Dic., 1992): 65-102.

Oscar Zelaya, "Tipificación del Grupo Social Dominante en el Antiguo Departamento de Tegucigalpa, 1839-1875." Tesis de Licenciatura, Carrera de Historia, UNAH, 1992.

Dario A. Euraque, "San Pedro Sula, Actual Capital Industrial de Honduras: Su Trayectoria entre Villorrio Colonial y Emporio Bananero, 1536-1936," Mesoamérica, No. 26, Vermont-Guatemala, (Dic., 1993): 217-252.

Ibrahim Gomero Idiaquez, Un Canto a San Pedro Sula (San Pedro Sula: Centro Editorial, 1993).

Mario Posas, Breve Historia de la Ciudad de La Ceiba (La Ceiba: Alcaldía Municipal de La Ceiba, Secretaria de Cultura, 1993).

José Iván Herrera, "Los Ciclos de Reproducción del Capital Bananero y Su Influencia en el Subdesarrollo de Honduras. 1870 Hasta el Presente," Economía Política, No. 28 (1993): 37-68.

Mario Posas, Breve Historia de La Ciudad de Olanchito (Olanchito: Alcaldía Municipal de Olanchito, 1994).

Leticia Oyuela, Un Siglo en la Hacienda: Estancias y Haciendas en la Antigua Alcaldía Mayor de Tegucigalpa (1670-1850) (Tegucigalpa: Banco Central de Honduras, 1994a).

Juan Ramón Funez H., Historia de San Jorge de Olanchito (Tegucigalpa: Editorial Universitaria, 1995).

Marvin Barahona, La Alcaldía Mayor de Tegucigalpa bajo el Régimen de Intendencias (1788-1812), Tegucigalpa, Instituto Hondureño de Antropología e Historia, 1996.

Mario Bueso Yescas, Santa Rosa de Los Llanos: Cuna de la Republica (San Pedro Sula: Graficentro Editores, 1996).

Darío A. Euraque, El Capitalismo de San Pedro Sula y la Historia Política de Honduras, 1870-1972 (Tegucigalpa: Editorial Guaymuras, 1996).

Carlos Elvir Aceituno, Laberinto Dorado: San Juancito- La Rosario (San Pedro Sula: Centro Editorial, 1996).

Darío A. Euraque, "Un Siglo en la Hacienda: Estancias y Haciendas en la Antigua alcaldía Mayor de Tegucigalpa, 1670-1850," Paraninfo, No. 9 (Julio 1996): 173-182.

Taylor Mack, "Ephemeral Hinterlands and the Historical Geography of Trujillo, Honduras, 1525-1950,” Ph.D diss., Louisiana State University, 1997.

Kevin Ruben Avalos y Joaquín Pagan Solórzano, "Inventario de Padrones, Cuadros Estadísticos y Matriculas de Varones Existentes en el Archivo Nacional de Honduras," YAXKIN, Revista del Instituto Hondureño de Antropología e Historia, Volumen XVI,
Nos. 1 y 2 (Diciembre 1997): 138-157

Ángela Maria Stassano Raquel, Adobe, Madera y Ladrillo en la Arquitectura de San Pedro Sula: Un Vistazo Actual a la Arquitectura Creada entre 1900 y 1950 (Tegucigalpa: Editorial Transamerica S.A., 1997).

Marcos Carias, Crónicas ty Cronistas de la Conquista de Honduras (Tegucigalpa: Editorial Universitaria, 1998).

John Soluri, "Landscape and Livelihood: An Agroecological History of Export Banana Growing in Honduras, 1870-1975," Ph.D diss., The University of Michigan, 1998. Luis Pedro Taracena, Ilusión Minera y Poder Político: La Alcaldía Mayor de Tegucigalpa, Siglo XVIII (Tegucigalpa: Editorial Guaymuras, 1998).

Antonio Canelas Díaz, La Ceiba, Sus Raíces y Su Historia (1810-1940) (La Ceiba: Tipografía Renacimiento, 1999).

Rafael Ángel Elvir. La Villa de la Cruz en la Historia, Llamada Tela desde 1829 (San Pedro Sula: Central Impresora, 2000).

Juan José Herrera Bobadilla, Un Baúl de Recuerdos (San Pedro Sula: Centro Edito rial, 2001)

Jordan Dym, "A Sovereign State of Every Village: City, State and Nation in Independence era Central America, ca. 1760-1850," Ph.D diss., New York University, 2000.

Douglas A. Tompson, "Frontiers of Identity: The Atlantic Coast and the Formation of Honduras and Nicaragua, 1786-1894," Ph.D diss., University of Florida, 2001.

Juan Blas Galeas, San Esteban: Una Aproximación a Su Historia (Tegucigalpa: Editorial Guaymuras, 2001).

Mark A. Bonta, "Mapping Enredos of Complex Spaces: A Regional Geography of Olancho, Honduras," Ph.D diss., Louisiana State University, 2001.

Antonio Canelas Díaz, El Estrangulamiento Económico de La Ceiba, 1903-1965 (La Ceiba: Editorial Pro-Cultura, 2001)

Julio Escoto, Compilador, Imágenes de San Pedro Sula (San Pedro Sula: Centro Editorial, 2002).

Fernando Cruz Sandoval, "A 200 Años de Historia Garifuna en Honduras: Bases para Una Periodizacion," YAXKIN, Revista del Instituto Hondureño de Antropología e Historia, Volumen XXI (2002): 89-111.

José Iván Herrera, "Sitios, Estancias y Haciendas Coloniales en Olanchito," Ciencia y Tecnología, Dirección de investigación Científica de la Universidad Nacional Autónoma de Honduras, No. 13 (Diciembre 2003): 23-43.

Darío A. Euraque, "Procesos e Infraestructuras de la Hondureñidad en el Siglo XX," en Honduras. Sucesos del Siglo XX, Compilador, Julio Escoto (San Pedro Sula: Centro Editorial, 2003), pp. 35-57.

Oscar Acosta, Selección, Elogio de Tegucigalpa, Edición Conmemorativa [Primera edición, 1978] (Tegucigalpa: Alcaldia Municipal del Distrito Central, 2003).

Leticia Oyuela, Esplendor y Miseria en la Mineria en Honduras (Tegucigalpa: Editorial Guaymuras, 2003).

Gladis Menjivar y Marysabel Zelaya, "Los Censos de Población en Honduras," en Población y Desarrollo: Argonautas y Caminantes, Maestría de Demografia Social, Compilador (Tegucigalpa: Postgrado Latinoamericano en Trabajo Social, UNAH, 2004): 15-20.

Rolando Sierra Fonseca, "El Estudio de las Migraciones en Honduras," en Pobla ción y Desarrollo: Argonautas y Caminantes, Maestría de Demografía Social, Compilador (Tegucigalpa: Postgrado Latinoamericano en Trabajo Social, UNAH, 2004): 61-62

David A. Figueroa Toruno y Elvis Geovany Trejo Teruel, "Las Encuestas Sociodemográficas en Honduras," en Población y Desarrollo: Argonautas y Caminantes, Maestría de Demografia Social, Compilador (Tegucigalpa: Postgrado Latinoamericano en Trabajo Social, UNAH, 2004): 23-29.

David J. Dodds, "Población y Detección de Cambios Ambientales por Satélite en la Costa Norte de La Mosquitia, 1960-1996," en Memoria, VII Seminario de Antropología de Honduras "Dr. George Haseman", Instituto Hondureño de Antropología e Historia (IHAH) (Tegucigalpa: IHAH, 2004): 347-371.

Rebecca Becerra, Memorias sobre el Barrio Abajo de Tegucigalpa (Tegucigalpa: Mujeres en el Arte, 2004).

Darío A. Euraque, "Negritud Garifuna y Coyunturas Políticas en la Costa Norte de Honduras, 1940-1970," en Darío A. Euraque Conversaciones Historicas con el Mestizaje en Honduras y su Identidad Nacional (San Pedro Sula: Centro Editorial, 2004), pp. 163-246.

Elizet Payne Iglesias, "El Puerto de Trujillo. Espacio, Economía, y Sociedad (17801870),” Tesis Doctoral, Universidad de Costa Rica, 2005. 